\title{
Journal of Strength and Conditioning Research
}

\section{Reliability and comparability of the accelerometer and the linear position measuring device in Resistance Training \\ --Manuscript Draft--}

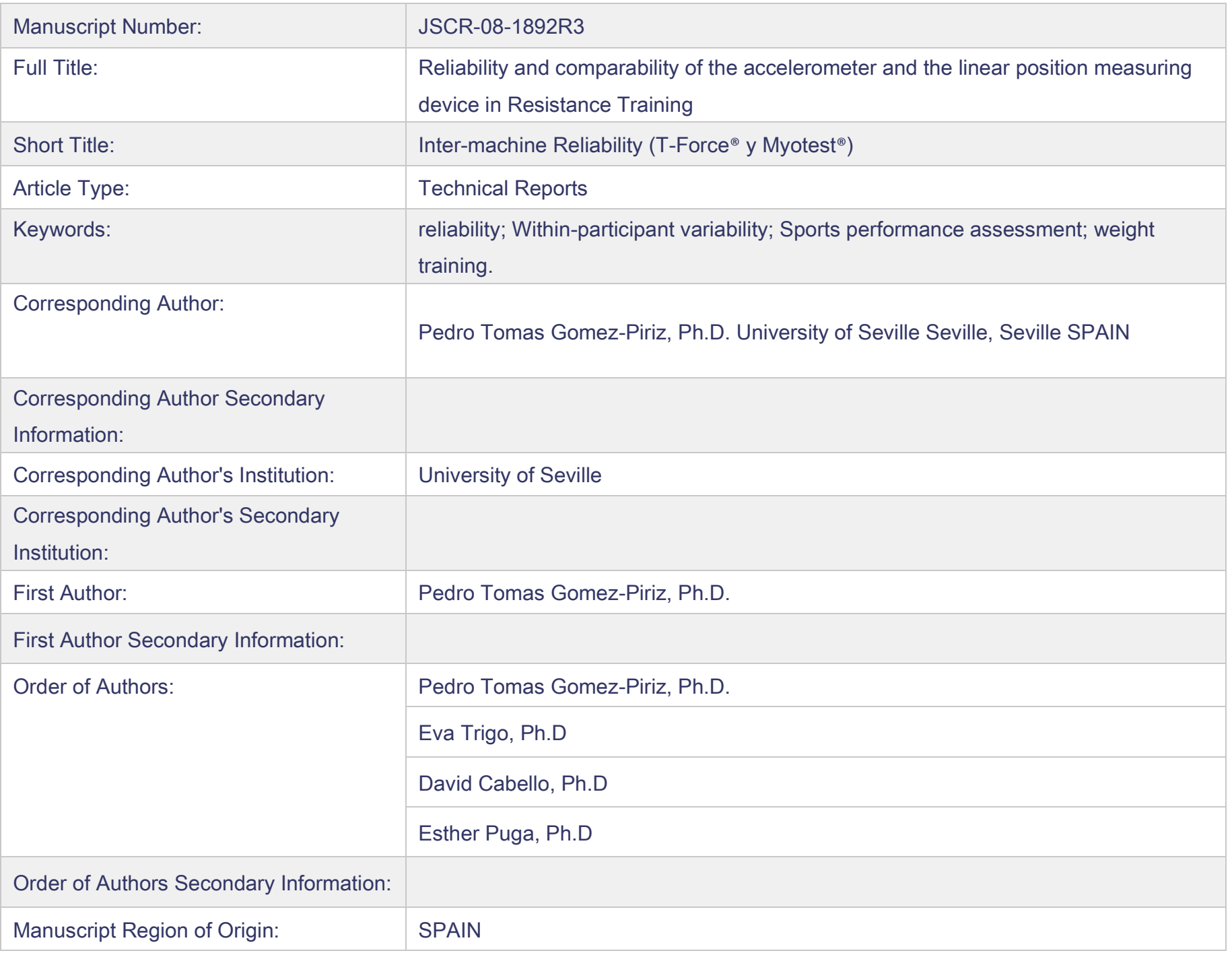



devices used to measure the common variables in sports performance. Repeatability conditions were established by creating a similar set of conditions under which the measurements were taken from both devices. Objectives: To demonstrate the reliability between two devices in a bench press movement: the linear position measuring device (LPM) isoinertial dynamometer (T-Force) and 2; the 3-D (Myotest) accelerometer (AC). To compare the existing correlations between maximum velocity, maximum estimated strength and peak power estimate variables in the bench press exercise. Method: 40 bench press exercise trials were analysed simultaneously, performed by three different subjects (age: $26.74 \pm 1.2$ years, height: $175.74 \pm 4.04 \mathrm{~cm}$, weight: $78.7 \pm 3.35 \mathrm{~kg})$ at maximum velocity $(25 \mathrm{~kg}$ additional load). Statistical Analyses: Three simple linear regression models were developed, supplied by the linear position measuring device (LPM) on the basis of the accelerometer's (AC) data. The assumption of independence of errors was compared by means of the Durban-Watson test and partial autocorrelation coefficients were established at an overall $p<0.05$ significance level. Results: It has not been possible to confirm the presence of a general correlation between the measurements of both devices. Regarding the assumption of independence of errors, the presence of generalised autocorrelation was confirmed. Linear regression analysis revealed an inter-machine correlation in one of the nonconclusive cases, (Peak Power) variable and subject $1, r(10)=0.640, p=0.024$. No partial autocorrelation was found. Practical applications: The devices should not be used interchangeably as instruments to assess and monitor resistance training. The AC device revealed higher and more disperse values than the LPM device. 
Title: Reliability and Comparability of the Accelerometer and the Linear Position Measuring Device in Resistance Training

Running head: Inter-machine Reliability (T-Force $\AA$ y Myotest ${ }^{\circledR}$ )

Authors: Pedro Tomas Gomez-Piriz*, Eva Trigo Sanchez*, David Cabello Manrique $^{\star *}$ and Esther Puga Gonzalez ${ }^{* *}$.

* University of Seville, Spain.

${ }^{* *}$ University of Granada, Spain.

Corresponding author: Pedro Tomás Gómez-Píriz

Mailing address: Department of Physical Education and Sport, University of Seville. C/Pirotecnia s/n. 41013. Sevilla. Spain

Telephone: 0034954420461

Fax: 95.455 .59 .85

Email address: ptgomez@us.es 
1

\section{INTRODUCTION}

2 The purpose of this technical report was to examine the measurable properties of two

3 commercially available devices routinely used in the field of sports training: it sets out 4 to compare their post-processing characteristics highlighting their differences and 5 advising specialist professionals against their interchangeable use when assessing and 6 monitoring sports performance.

7 In this instance, the reliability of measurement refers to the reproducibility of results in

8 repeated trials carried out with both devices. Clarification of this issue allows for a 9 greater understanding of the researchers' theory of reliability, which helps reduce the 10 incidence of inappropriate analyses in the literature of Sports Science (1).

11 Several studies have examined the concept of inter-machine reliability between devices accelerometer (model 3140, ICS Sensors, USA) and a cable-extension position transducer (model PT5DC, Celesco, USA) in a simulated mechanical laboratory setup (2). Results revealed a coefficient of variation in the repeated measures in just one of the measured variables analyzed. In turn, greater data variability was revealed when assessing athletes' performance (3). With the same objective in mind, the same study analyzed the values obtained from three models of accelerometer (Actical, Actigraph and RT3) and from the results obtained, it was recommended that future studies should focus on understanding why accelerometers designed to measure the same variables gave such different results. Furthermore, the need to control the parameters for measurement accuracy in successive research was emphasized (5). Demonstrating that data obtained from both devices commonly used in training should be the same is therefore a key issue. Consequently, this aspect is examined in this report by comparing two measuring devices:

A) The inertial dynamometer, (T-Force System Ergotech, Murcia, Spain) referred to in this report as LPM, is a device routinely used in the measurement and monitoring of training using additional loads and has recently been employed in several research studies $(4,6,7,19)$.

B) The Accelerometer 3-D Myotest Sport S4P model (Myotest SA, Sion, Switzerland), which here is referred to as AC. This device is also currently widely used to monitor and measure diverse force values. It has recently been used for varied functions in this 
1 field that have proved its worth (8, 9, and 10). Comstock et al. (11) verified the

2 concurrent validity of this device (AC) when compared to a computerized linear

3 transducer and force platform system (Celesco linear transducer of the directly

4 interfaced BMS system, Ballistic Measurement System Innervations Inc, Fitness

5 Technology force plate, Skye, South Australia, Australia) finding a strong, positive

6 correlation and concluding that when fixed on the bar in the vertical axis, the Myotest is

7 a valid field instrument for measuring force and power.

8 Assessment of the validity and reliability between the AC device and a photoelectric 9 cells measurement system (Optojump, Microgate, Bolzano, Italy) was expanded upon in a study (12) and determined the need for additional analysis to prove the latter's reliability. The authors concluded that $\mathrm{AC}$ is a valid and reliable tool for assessing vertical jump height performance but advised that there should be no cross-over of data from one device to the other, due to the systematic overestimation of jumping height by the $\mathrm{AC}$ device. between the AC device and other linear position measuring devices for the variables of maximum velocity, force and peak power in the bench press exercise. The authors of this study concluded that the algorithms used by both devices could be the possible source of the lack of data correlation. These three variables are used in several studies concerned with training assessment $(4,5,6,7,8,11$ and 19)

The practical questions addressed in our study therefore relate to the measurement of kinematic variables using different devices, LPM (T-Force) and AC (Myotest). Do these produce the same results? Can the devices be interchanged indiscriminately? How can the results of each device be isolated given the existing autocorrelation of data produced by each athlete?

Sufficient objective grounds exist to raise doubts about the measurement of the same variables by these two devices, which increase when it comes to assessing athletes. For this reason the following aims were established:

-To compare data obtained by both devices and observe their evolution, establishing strategies in the test design to control the presence of autocorrelation among the output variables between trials carried out by the athletes. 
1 -To correlate the results obtained by both devices and establish the relevant parameters

2 to assess and monitor the training session.

3 The general hypothesis of the study was based on the theory that different

4 measurements carried out in identical conditions by the two devices should produce the

5 same results. Prediction models were defined by observable parameters, in this instance,

6 based on standard variables in resistance training when performing the bench press

7 exercise.

8 Finally, the following statistical assumptions were made:

9 1. The results obtained from both devices should allow the interreliability of both 10 devices to be demonstrated.

11 2. Autocorrelation is present in the different measurements taken from the subjects that interfere with obtaining inter-machine correlation.

3. Statistically significant correlation is present between results obtained from both devices, carried out under the research conditions outlined, between the variables of maximum velocity, estimated maximum force and estimated peak power when performing the bench press exercise.

\section{METHODS}

Experimental Approach to the Problem

The data from both tested devices were collected simultaneously. A) the linear position measuring device -LPM- (Isoinertial Dynamometer (Model TF-100, T-Force System Ergotech, Murcia, Spain) - a system that via a cable extension transducer translates movement generated (sampled at a frequency of $1000 \mathrm{~Hz}$ ) to the linear velocity with which it is displaced by means of calibration constant $\mathrm{K}=.4899$ (each device is factorycalibrated). The related software (T-Force v.2.28) calculates the kinematic variables via invariant statistical methodology obtained from the kinetic variables. B) The 27 accelerometer -AC, (3-D Myotest Sport S4P model, Myotest SA, Sion, Switzerland), 28 which calculates velocity, force and power resulting from the measurement of acceleration (time variation of velocity) computing the kinetic variables (MyotestPRO v.1.00.20995). The device receptor is very small (W x L x H: 54.2 x 1032.5 x 10.7mm) 
1 and weighs $58 \mathrm{~g}$. The rate of data acquisition is $200-500 \mathrm{~Hz}$, dependent on assessment

2 requirements, connected via a USB 2.0 interface cable.

3 Subjects

4 Three different athletes took part in this study (age: $26.74 \pm 1.2$ years, height: $175.74 \pm$ $54.04 \mathrm{~cm}$, weight: $78.7 \pm 3.35 \mathrm{~kg}$ ) performing a total of 40 bench press exercises (subject

$6 \quad 1: 12:$ subject 2: 12 and subject 3: 16)

7 All gave written consent for their voluntary participation in this research study. No 8 physical limitations, health problems, or musculoskeletal injuries that could affect 9 testing were found after a medical examination. The subjects had a continental breakfast 1090 minutes before the start of the session. None of the subjects were taking drugs, medications, or dietary supplements known to influence physical performance. All subjects in the study were in the pre-competition phase of their training, performing an average of 2 weight-training sessions per week. The study met the ethical standards of this Journal and was approved by the Ethics Committee of the University of Seville.

Procedures

All subjects performed the tests at the High Performance Sports Centre in Sierra Nevada (Granada: atmospheric pressure $1003 \mathrm{hPa}$, absolute, relative humidity: 93.3\%, elevation: $2320 \mathrm{~m}$ above sea level and temperature: $15^{\circ} \mathrm{C}$ ).

The trials were carried out on the bench press by an exercise that exercises the upper limbs. The subjects adopted the following starting position: lying supine on the bench, knees flexed and feet resting on the bench, elbows bent to $90^{\circ}$ and shoulders abducted to $90^{\circ}$. Grip width was assessed previously and enabled the aforementioned joint angles to be maintained at the starting position $(5,6 ; 13)$. The subjects were asked to perform a free-weight vertical lift, moving the barbell $(25 \mathrm{~kg})$ as fast as possible during the concentric phase. As this was a concentric phase the beginning was when the bar was at rest and the end was when the bar had been lifted to its greatest height. After a gradual warm-up, all the trials were performed at a frequency that included a three minutes rest period. The two devices employed in this comparative study were secured in accordance to specified instructions, on to the same lateral edge of the barbell. The following test variables were analyzed simultaneously: 
1 Peak Power (W), calculated from the force-velocity relationship registered during the 2 movement.

3 Maximum velocity $(\mathrm{cm} / \mathrm{s})$, obtained from the time-space variation.

4 Maximum Force $(\mathrm{N})$, calculated from the additional load-acceleration relationship

5 registered during the entire movement.

$6 \quad$ Statistical Analyses

7 Three simple lineal regression models were developed based on the three variables

8 supplied by the LPM (criterion variable) and on the basis of the AC data (predictor 9 variable).

10 The assumption of independence of errors was compared by means of the Durbin11 Watson test, due to its effect on the increase to error rate type 1. For the statistical 12 process control of possible autocorrelation, partial autocorrelation coefficients were 13 calculated in each instance, contemplating a single series produced from the measures 14 provided by both devices and a delay equal to the number of trials performed. Statistical 15 significance level was set at $\mathrm{p}<0.05$ for all tests. Additionally, the effect size obtained 16 in each concurring case was evaluated in accordance with levels proposed by Cohen 17 (14, 15). The data were smoothed using the software supplied as part of each device. 18 Data analysis was carried out using the Statistical Package for the Social Sciences 19 (SPSS Inc., version 18, Chicago).

\section{RESULTS}

21 A higher average value was detected in the majority of the variables measured by the 22 AC device compared to the LPM.

23 The mean and standard deviations for each of the measurements analyzed in the series 24 of three tests are set out in Table 1. The average measurements supplied by the AC 25 device are higher in the majority of cases compared to the average LPM measurements, 26 with the exception of the maximum force variables of subject 3 and the maximum 27 velocity variables of subject 1 . At the same time, the standard deviations of the AC 28 measurements are higher than those of the LPM on all occasions except one; the measurement of maximum force of the third subject. 
1 In Table 2 the statistical values of the Durbin-Watson test are shown for each and every

2 one of the trials. The only instance, in which this is clearly not the case is in the

3 measurement of maximum force for subject 1; in four instances, the test data are not

4 conclusive, thereby indicating that autocorrelation may be present. In the remaining

5 cases the presence of autocorrelation is apparent.

6 However, not even in the aforementioned instances of possible increase in error rate

7 type 1 did significant correlation prevail insofar as the measurements provided by both 8 devices were concerned. In the non-conclusive data cases, only one statistically 9 significant correlation was found and with a large effect size; the peak power measurement of subject $1, r(10)=0.640, p=0.024$.

In the remaining instances, autocorrelation aside, only two statistically significant correlations were found and with a large effect size; the measurement of peak power and velocity of the third subject, $r(14)=0.670 \mathrm{p}=0.005$, in both cases.

The high correlation factor between these measurements for subject 3 cannot be considered as replicated by the measurement of maximum force in subject 1, given the existence of autocorrelation between the error terms of independent variables.

$* * * * * * *$ Table 2 about here $* * * * * * *$

In order to obtain additional support for the absence of positive correlation between both devices, partial autocorrelation was calculated for the statistical control of the possible existence of autocorrelation between the measurements obtained from a single subject using the same device. Table 3 gives these partial autocorrelations and their respective confidence intervals.

As can be seen, the majority of the autocorrelation values proved negative, including some of those corresponding to measurements where a positive statistically significant correlation was found by means of linear regression analysis. This indicates that an increase in the data provided by one device occurs as a decrease in the data provided by the other, meaning that their similarity cannot be endorsed. This notion is also supported by the fact that the only partial positive autocorrelation present is very small. In any event, none of the correlations surpassed the $95 \%$ confidence interval, so that they cannot be said to be statistically significant.

$* * * * * * *$ Table 3 about here $* * * * * * *$ 
1

\section{DISCUSSION}

2 The reliability of data obtained between measuring devices is an ongoing and as yet 3 unresolved issue, which, as highlighted above (1), continues to be of key concern to the 4 scientific community. This, in conjunction with the observation made (2) that the main 5 variability of data arises when measuring individual athlete performance, poses an 6 additional challenge. This factor has been considered of great importance when 7 designing and subsequently analyzing the statistical data in this report. The control of 8 characteristics derived from the error produced by data dependence for the same subject 9 in successive trials has enabled results to be obtained in keeping with the purpose of this technical report.

This assertion results from the non-existence of widespread high positive correlation between the two sets of data obtained from the two devices under examination, in which the presence of a substantial low and even negative correlation was apparent. This has also been evident in other inter-machine comparability studies that have been carried out $(4,16,2,3)$. Furthermore, we concluded that a strong (11), positive correlation existed between the AC device and a computerized linear transducer and force platform system (Celesco linear transducer of the directly interfaced BMS system, Ballistic Measurement System Innervations Inc, Fitness Technology force plate, Skye, South Australia, Australia)

The values obtained in our study denote how the AC device shows higher average values and a higher standard deviation (Table 1) with the exception of two instances; the peak power measurement of the third subject and the velocity maximum measurement of the first. This leads us to deduce that when employing this device, the values measured will be higher, as was the case in the data obtained regarding the overestimation of jumping height by the AC device when compared to another linear position measuring device (12). Obtaining higher values, as well as greater dispersion in AC compared to LPM, is yet a further reason showing that data obtained from both devices should not be used simultaneously, so confirming the aims of this Report. Moreover these higher values will therefore be less accurate, since the data distribution registered higher dispersion when compared to data obtained from the LPM device. On the other hand, the LPM device registered lower average values with less dispersion, which may imply greater accuracy. 
1 As to the assumption of independence of errors in accordance with the Durbin-Watson

2 test (not found to be applied in previous cited studies), in only one instance was this not

3 found; the measurement of maximum force for subject 1, although in four other

4 instances the test data was non-conclusive, which indicated that autocorrelation could be

5 present. This analysis allowed for the possible existence of inter-machine correlation

6 when this is due to the autocorrelation of the subjects in successive trials. Only one

7 statistically significant correlation was found, with a large effect size and with no

8 autocorrelation - the peak power measurement of subject $1, r(10)=0.640, p=0.024$,

9 although the data revealed a non-conclusive value of independence which makes replication of this affirmation for all the results obtained impossible. In order to confirm the analysis, partial negative autocorrelation was found and only a very small positive autocorrelation but none of these autocorrelations surpassed the $95 \%$ confidence interval limits, which meant that they could not be considered to be of statistical significance (Table 3).

It could also be confirmed that on measuring the test variables with the AC device, there is an increase in the value of uncertainty of the measurement (a concept that represents a quantitative measure of quality of the result of measuring, enabling the results obtained from the devices to be compared with other results, references, specifications or rules) which is, referred to as typical value of uncertainty Type A by the Spanish Center of Metrology (17). This parameter is associated with the result of a measurement which characterizes dispersion of the values that can be reasonably attributed to each measure. This is apparent in Figure 1, in which the values of each variable measured by each device are reflected and the relative distance to an established value from an absolute value of zero, which must correspond with the real value of the measurand.

Considering these values (Figure 1), in the maximum velocity variable the results from the three trials obtained from the $\mathrm{AC}$ device are very different to those obtained from the LPM device. In the central image (maximum force) and on the basis of the values of this variable, this difference occurs in 6 trials. Finally, and as a result of the estimate from the algorithms of each device, in the third variable (peak power) there is a greater dispersion in the AC device compared to that of the LPM device for the two groups of trials related to the previous variables, as was to be expected given the calculations made. 
1 The algorithms could be the cause of these issues, particularly in the case of the AC

2 device, as has been revealed by other studies carried out on this device $(12,13)$.

3 The uncertainty of the results of a measurement reflects the impossibility of knowing

4 the exact value of the measurand. This could be due to: a finite resolution of the 5 measuring device or its discrimination threshold, inexact values of measurement

6 standards; the inexact values of the patterns and other parameters drawn from outside

7 sources and used in the data processing algorithms (17).

$* * * * * * *$ Figure 1 about here ${ }^{* * * * * * *}$

It should be noted that possible differences in the technical characteristics of both devices, for example the different velocities of data collection (LPM; $1000 \mathrm{~Hz}$ compared to AC; 200-500 Hz) may explain these variations. In this respect, the relevant aspects regarding the existing calibration specifications of the LPM device have to be considered. These are obtained by a direct comparison with the inexact values of measurement standards or the certified reference materials. Due to the presence of data variability obtained from testing a standardized bout of treadmill walking (16), the authors of that study reached the conclusion that appropriate calibration protocols are needed to ensure the reliability of the measures analyzed in four different types of accelerometer (CSA/MTI, Biotrainer Pro,Tritrac-R3D, and Actical). According to the Spanish Center of Metrology (18), the existence of the calibration protocol of a device demonstrates the traceability to which it is subject, guarantees that its readings are compatible with other measurements, determines the accuracy of the readings of the monitoring device and establishes its reliability.

This study does not set out to question the accuracy of the results obtained from both devices, since this is not its objective. That is the objective of the organizations that deal with this matter, as is the comparison of the accepted reference value (true or actual value) for each of the variables studied.

\section{PRACTICAL APPLICATIONS.}

The purpose of this technical report was to examine the reliability between both monitoring devices by comparing the values of one over the other. The nonconfirmation of correlation was established between the T-Force device (LPM) and the 
1 Myotest Sport accelerometer (S4P model) (AC), meaning that the data obtained from one could not be predicted on the basis of the data obtained from the other, which in turn means that the statistical assumption could not be confirmed. Statistical hypothesis 3 established the presence of a statistically significant correlation between the results obtained from both devices, under the research conditions established, between the variables of maximum velocity, estimated maximum force and estimated peak power when performing the bench press exercise. Therefore, we conclude that it is necessary to advise against the interchangeable use of both devices as instruments to assess and monitor resistance training. It is recommended that the same device is always employed and that the data obtained from the same is used as the sole means of comparing data in subsequent training sessions. In this way, confusion as to the interpretation of any increase or decrease that may be manifested in the results, key to monitoring the results obtained in training, would be avoided, as would any confusion with regards to the input of reference values in successive testing. These conclusions have been reached taking into account the control of independence of errors between tests carried out on each of the subjects who participated. The data revealed the presence of a generalized autocorrelation by means of the assumption of independence of errors in accordance with the Durbin-Watson test. Independence of errors between the results of the same subject was only apparent in one instance (subject 1 -maximum force variable), which permits us to assert that that the data obtained from the athletes were characterized by a generalized autocorrelation, thereby confirming statistical assumption 2. For future tests, it is recommended that design strategies that reflect error control resulting from the data dependence factor be applied when measuring an athlete's performance.

The AC device (Myotest) revealed higher and more disperse values than the LPM device (T-Force). This was confirmed in 16 of the 18 cases considered, although this was not the case for the peak power measurement of the third subject and the maximum velocity measurement of the first. Evidence therefore points to an overestimation and greater dispersion and a lack of accuracy in the data given by the AC device when compared to the LPM device.

Bearing all the above in mind, since there are risks in the concordance of the data between the two devices, trainers and athletes are recommended to take special care when using the variables power peak, maximum force and maximum velocity, controlling such values and comparing them only with the values obtained from the 
1 same device. Finally since both devices are used habitually, we would recommend that

2 all results are measured, controlled and compared using the same device. Mixing the

3 data of measurements obtained indiscriminately from one or the other can falsify the

4 results of training

5

6 References:

71 Hopkins, W. G. Measures of Reliability in Sports Medicine and Science. Sports Med; $830(1): 1-15,2000$.

92 Jidovtseff B, Croisier JL, Lhermerout C, Serre L, Sac D and Crielaard JM. The 10 concept of iso-inertial assessment: reproducibility analysis and descriptive data. Isokinetics and Exercise Science, 14, 1, 53-62, 2006.

3 Esliger, DW and Tremblay, MS. Technical reliability assessment of three accelerometer models in a mechanical setup. Medicine \& Science in Sports \& Exercise, 38, 12, 2173-2181, 2006.

4. Garcia-Pallares J, Lopez-Gullon JM, Muriel X, Diaz A \& Izquierdo M. Physical fitness factors to predict male Olympic wrestling performance. Eur J Appl Physiol 111:1747-1758, 2011. DOI 10.1007/s00421-010-1809-8

5 Bosquet, L, Porta-Benache, J and Blais, J. Validity of a commercial linear encoder to estimate bench press $1 \mathrm{RM}$ from the force-velocity relationship. Journal of Sports Science \& Medicine, Vol. 9 Issue 3, 459-463, 2010.

6 Sanchez-Medina L, Pérez, CE and Gonzalez-Badillo JJ. Importance of the Propulsive Phase in Strength Assessment. Int J Sports Med; 31: 123-129, 2010.

7 Gonzalez-Badillo, JJ and Sanchez-Medina, L. Movement Velocity as a Measure of Loading Intensity in Resistance Training. Int J Sports Med; 31: 347-352, 2010.

8 Kraemer, W. Construct validity of the Myotest ${ }^{\circledR}$ in measuring force and power production. Abstract $32^{\text {nd }}$ National Conference 6 Exhibition (NSCA, Las Vegas), p.26, 2009.

9 El Hage, R, Zakhem, E. Moussa, E and Jacob C. Acute effects of heavy-load squats on consecutive vertical jump Performance. Science \& Sports 26, 44-47, 2011. 
110 Hasegawa, H, Yamauchi, T, Kawasaki, T, Adachi, T and Yamashita, M and

2 Nakashima, N. Effects of plyometric training using a portable self-coaching system on

3 Running performance and Biomechanical variables in jump Exercises. J Strength Cond

4 Res, 25, supplement 1, 2011.

511 Comstock, BA, Solomon-Hill, G, Flanagan, SD, Earp, JE, Luk, H-Y, Dobbins, KA,

6 Dunn-Lewis, C, Fragala, MS, Ho, J-Y, Hatfield, DL, Vingren, JL, Denegar, CR, Volek,

7 JS, Kupchak, BR, Maresh, CR, and Kraemer, WJ. Validity of the myotest (R) in

8 measuring force and power production in the squat and bench press. $J$ Strength Cond $9 \quad \operatorname{Res} 25$ (8): 2293-2297, 2011.

1012 Casartelli, N, Müller, R and Maffiuletti, N. Validity and reliability of the Myotest accelerometric system for the assessment of vertical jump height. J Strength Cond Res, 24(11)/3186-3193, 2011.

13 Jidovtseff, B, Crielaard, JM, Cauchy, S and Croisier, JL. Validity and reliability of an inertial dynamometer using accelerometry. Science \& Sports, 23, 94-97, 2008.

14 Cohen, J. Statistical power analysis for the behavioral sciences (2nd Ed.). Hillsdale, NJ: Lawrence Erlbaum Associates, 1988.

15 Cohen, J. A power primer. Psychological Bulletin, 112, 155-159, 1992.

16 Welk, GJ, Schaben, JA and Morrow JR. Reliability of accelerometry-based activity Monitors: a generalizability study. Medicine \& Science in Sports \& Exercise, 36, 9, 1637-1645, 2004.

17 Centro Español de Metrología (CEM). Vocabulario Internacional de Metrología. (VIM) ( $3^{\mathrm{a}}$ ed.) Madrid. (www.cem.es), 2008.

18 Centro Español de Metrología (CEM). Metrología Abreviada (3 ${ }^{\mathrm{a}}$ ed.) Madrid. (www.cem.es), 2008b.

19 Sanchez-Medina L and Gonzalez-Badillo JJ. Velocity Loss as an Indicator of Neuromuscular Fatigue during Resistance Training. Medicine \& Science in Sports \& Exercise, 1725-1734 (Accepted for publication February 2011. DOI: 10.1249/MSS.0b013e318213f880) 
1 Figure Legends:

2

3 Figure 1. Data distribution of the variables analyzed from an absolute zero point by AC 4 and LPM.

5

6 Table Legends:

7

8 Table 1. Descriptive Statistics of the three measurements recorded by each device used 9 in each of the test series.

10 Table 2. Empirical and theoretical (interval) values of the statistician Durbin-Watson 11 and simple linear regression analysis data for each measurement with the data collected 12 by the Linear Position Measuring Device (LPM) as the criterion variable and the data 13 from the accelerometer as the predictor variable.

14 Table 3. Partial autocorrelations for the different measurements obtained from each 15 subject. 
Table 1. Descriptive Statistics $(\mathrm{M}=$ mean/average and $\mathrm{SD}=$ Standard deviation $)$ of the three measurements recorded by each device used in each of the test series.

\begin{tabular}{cccccccc}
\multirow{2}{*}{ Subjects } & & \multicolumn{2}{c}{ Peak Power } & \multicolumn{2}{c}{ Max. Strength } & \multicolumn{2}{c}{ Max. Velocity } \\
& & $M$ & $S D$ & $M$ & $S D$ & $M$ & $S D$ \\
\hline \multirow{2}{*}{1} & LPM & 920.270 & 62.750 & 408.390 & 17.770 & 225.210 & 8.460 \\
& AC & 1169.830 & 316.050 & 559.170 & 191.700 & $\underline{215.170}$ & 32.710 \\
\hline \multirow{2}{*}{2} & LPM & 655.170 & 46.540 & 346.980 & 12.130 & 188.670 & 8.610 \\
& AC & 780.580 & 256.260 & 355.670 & 27.290 & 220.250 & 74.200 \\
\hline \multirow{3}{*}{3} & LPM & 410.810 & 45.220 & 322.260 & 42.750 & 129.450 & 20.550 \\
& AC & 462.810 & 153.270 & $\underline{307.940}$ & $\underline{20.540}$ & 148.190 & 42.530 \\
\hline \multicolumn{3}{c}{ AC = Acelerometer; LPM= Linear Position Measuring Device }
\end{tabular}

$\mathrm{AC}=$ Acelerometer; LPM= Linear Position Measuring Device 
Table 1. Empirical and theoretical (interval) values of the statistician Durbin-Watson ${ }^{1}$ and simple linear regression analysis data for each measurement with the data collected by the Linear Position Measuring Device (LPM) as the criterion variable and the data from the accelerometer as the predictor variable.

\begin{tabular}{ccccccccc} 
Subjets & & $D-W$ & Interval & $b$ & $\beta$ & $t$ & $d f$ & $p$ \\
\hline \multirow{3}{*}{1} & Max. Strength & $0.700^{*}$ & & 0.010 & 0.140 & 0.450 & 10 & 0.663 \\
& Peak Power & $1.170^{?}$ & $0.810-1.580$ & 0.130 & 0.640 & 2.660 & 10 & $0.024^{*}$ \\
& Max Velocity & $1.250^{?}$ & & -0.010 & -0.040 & -0.120 & 10 & 0.904 \\
\hline \multirow{2}{*}{2} & Max. Strength & $1.490^{?}$ & & -0.140 & -0.320 & -1.080 & 10 & 0.304 \\
& Peak Power & $1.570^{?}$ & $0.810-1.580$ & -0.050 & -0.250 & -0.820 & 10 & 0.429 \\
& Max. Velocity & 2.060 & & -0.040 & -0.310 & -1.020 & 10 & 0.333 \\
3 & Max Velocity & 1.580 & & 0.020 & 0.010 & 0.037 & 14 & 0.971 \\
& Peak Power & 1.690 & $0.980-1.540$ & 0.200 & 0.670 & 3.350 & 14 & $0.005^{*}$ \\
& Max. Velocity & 2.130 & & 0.320 & 0.670 & 3.350 & 14 & $0.005^{*}$ \\
\hline
\end{tabular}

* Statistically significant autocorrelation; ? non-conclusive data. 
Tabla 1. Partial autocorrelations for the different measurements obtained from each subject.

Peak Power Max. Strength Max. Velocity

\begin{tabular}{llll}
\hline Subject 1 & -0.180 & -0.110 & -0.180 \\
Subject 2 & -0.350 & -0.290 & -0.290 \\
Subject 3 & -0.010 & 0.050 & -0.070 \\
\hline
\end{tabular}


Power Peak (w) $+A C$ - LPM

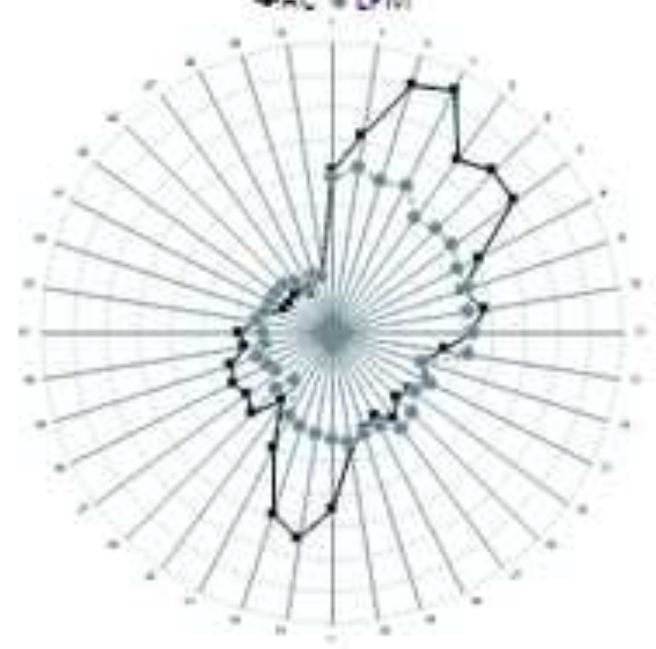

Force Max. (N)

$+A C+L F M$

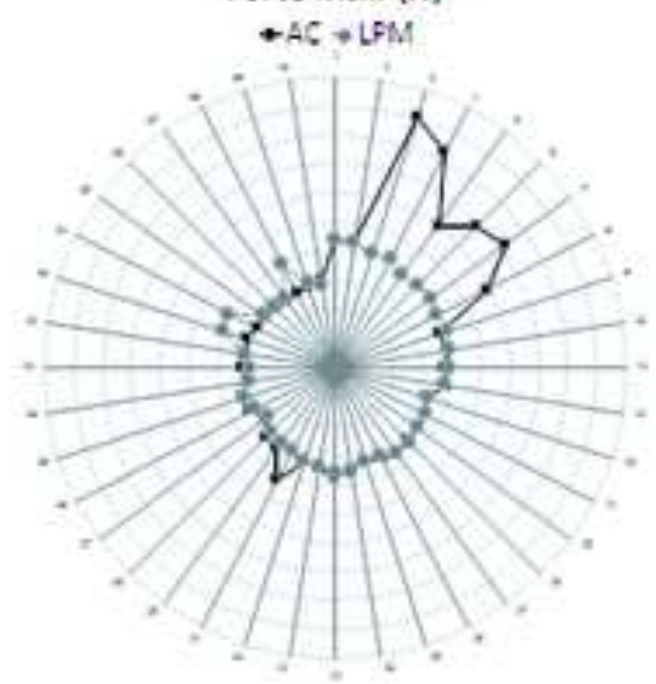

Max, velocity $(\mathrm{cm} / \mathrm{s})$

$\rightarrow A C+L P M$

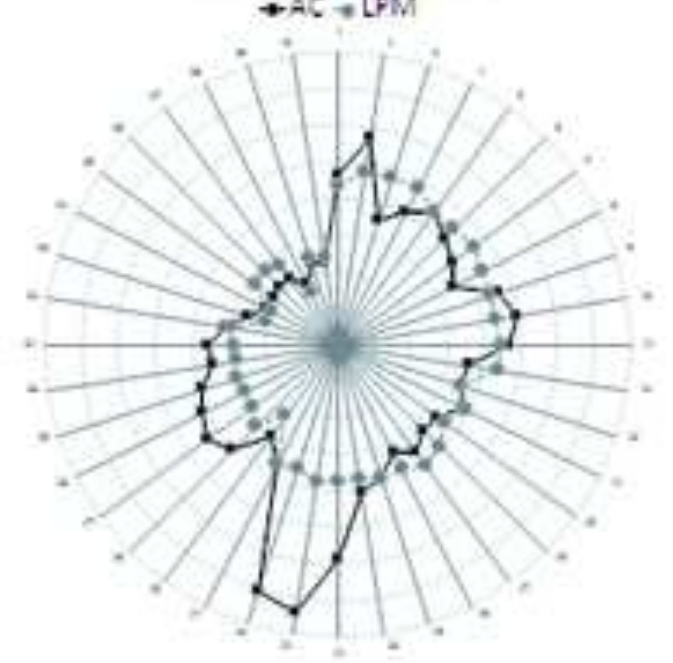

